\title{
SOME EXPONENTIAL MOMENTS OF SUMS OF INDEPENDENT RANDOM VARIABLES ${ }^{1}$
}

BY

\author{
J. KUELBS 2
}

\begin{abstract}
If $\left\{X_{n}\right\}$ is a sequence of vector valued random variables, $\left\{a_{n}\right\}$ a sequence of positive constants, and $M=\sup _{n>1}\left\|\left(X_{1}+\cdots+X_{n}\right) / a_{n}\right\|$, we examine when $E(\Phi(M))<\infty$ under various conditions on $\Phi,\left\{X_{n}\right\}$, and $\left\{a_{n}\right\}$. These integrability results easily apply to empirical distribution functions.
\end{abstract}

1. Introduction. Let $B$ denote a real vector space, $\mathscr{B}$ a sigma-algebra of subsets of $B$, and $\|\cdot\|$ a seminorm on $B$. We say the triple $(B, \mathscr{B},\|\cdot\|)$ is a linear measurable space if (i) addition and scalar multiplication are $\mathscr{B}$ measurable operations on $B$, (ii) for all $t>0$ we have $\{x \in B:\|x\|<t\} \mathscr{B}$ measurable, and (iii) there exists a subset $F$ of the $\mathscr{B}$ measurable linear functionals on $B$ such that

$$
\|x\|=\sup _{f \in F}|f(x)| \quad(x \in B) .
$$

Examples of linear measurable spaces are readily available in probability theory and, of course, include the situation where $B$ is a real separable Banach space, $\mathscr{B}$ denotes the Borel subsets of $B$, and $\|\cdot\|$ is the norm on $B$. Another important example consists of $B=D[0, T]$ where $D[0, T]$ denotes the real-valued functions on $[0, T]$ which are right continuous on $[0, T]$ and have left-hand limits on $(0, T]$. In this case $\mathscr{B}$ consists of the minimal sigma-algebra making the maps $x \rightarrow x(t), 0<t<T$, measurable and the seminorm is the sup-norm, $\|x\|=\sup _{0<t<T}|x(t)|$. The analogous $D$-spaces of functions of several variables are also linear measurable spaces (see $\$ 4$ and [17] for further details and references).

Now assume $(B, \mathscr{B},\|\cdot\|)$ is a linear measurable space, $\left\{X_{n}\right\}$ a sequence of $(B, B)$ valued random variables, $\left\{a_{n}\right\}$ a sequence of positive constants, and define

Received by the editors May 26, 1976 and, in revised form, October 13, 1976.

AMS (MOS) subject classifications (1970). Primary 60B05, 60F05, 60F15, 60G15.

Key words and phrases. Multidimensional empirical distribution function, exponential inequalities, exponential moments of the supremum of partial sums.

'It is a pleasure to acknowledge a number of important comments by M. B. Marcus and W. A. Woyczynski which led to the revision of this paper.

2Partially supported by the United States Army under Contract No. DAAG29-75-C-0024 and NSF Grant MPS75-05855. 


$$
M_{r}=\sup _{n>r}\left\|\frac{X_{1}+\cdots+X_{n}}{a_{n}}\right\| \quad(r>1) .
$$

In this paper we examine when

$$
E\left(\Phi\left(M_{1}\right)\right)<\infty
$$

provided $\Phi$ is a finite, nonnegative, nondecreasing function on $[0, \infty)$ and $\left\{X_{n}\right\}$ and $\left\{a_{n}\right\}$ satisfy various conditions.

In case $\Phi$ is a function which does not grow too rapidly the application of Theorem 3.3 of [6] and the technique of Corollary 3.4 of [7] immediately yield results. To be precise, assume $\left\{X_{n}\right\}$ is a sequence of independent random variables and $\Phi(4 u)<c \Phi(u)$ for some $c>0$ and all $u \in[0, \infty)$. If $P\left(M_{r}<\infty\right)=1$ for any $r$ (and hence all $r$ ), then the results in [6] and [7] indicated above imply that (1.3) holds if and only if

$$
E\left[\Phi\left(\sup _{n}\left\|\frac{X_{n}}{a_{n}}\right\|\right)\right]<\infty .
$$

For example, if the sequence $\left\{X_{n}\right\}$ is i.i.d. and $E\left\|X_{1}\right\|^{p}<\infty(0<p<\infty)$, then $P\left(M_{1}<\infty\right)=1$ and

$$
E\left(M_{1}^{p^{\prime}}\right)<\infty
$$

for $p^{\prime}<p$ provided $a_{n}=\max \left\{n, n^{1 / p}\right\}$. That is, to verify (1.5) one simply checks (1.4) with $\Phi(u)=u^{p^{\prime}}$ and this is trivial. Hence in this case $M_{1}$ has moments of order $p^{\prime}$ for all $p^{\prime}<p$ whenever $E\left\|X_{1}\right\|^{p}<\infty$.

Another example that we will be particularly interested in will be the situation when $\left\{X_{n}\right\}$ is an i.i.d. sequence with $E\left\|X_{1}\right\|^{p}<\infty(p>2)$ and $a_{n}=\sqrt{2 n L L n}(n>1)$. Here $L x$ denotes $\log x$ for $x>e$ and 1 otherwise, and $L L x$ denotes the composite function $L(L x)$. Then, if $P\left(M_{1}<\infty\right)=1$, we have

$$
E\left(M_{1}^{p^{\prime}}\right)<\infty
$$

for $p^{\prime}<p$. To verify (1.6) one simply checks (1.4) with $\Phi(u)=u^{p^{\prime}}$.

If $\Phi(u)=\exp (u)$ we can apply Theorem 3.8 of [6] along with the technique in Corollary 3.4 of [7]. In this case the assumption on the individual summands is that

$$
\sum_{n=1}^{\infty} E\left\{\exp \left(\alpha \frac{\left\|X_{n}\right\|}{a_{n}} \log ^{1+\delta} \frac{\left\|X_{n}\right\|}{a_{n}}\right) 1_{\left\{\left\|X_{n}\right\|>a_{n} \cdot a\right\}}\right\}<\infty
$$

for some $a>0, \alpha>0$, and $\delta>0$. Then $P\left(M_{1}<\infty\right)=1$ implies there exists a $\beta>0$ such that

$$
E\left(\exp \left(\beta M_{1}\right)\right)<\infty
$$


However, in case $\Phi(u)=\exp \left\{A u^{2}\right\}$ the results mentioned above or those in [9, pp. 3-14] do not apply, and it is this situation that we examine here. An example of this type was established in [13] where it was proved that if $\left\{\varepsilon_{j}\right.$ : $j>1\}$ is an i.i.d. sequence such that $P\left(\varepsilon_{j}= \pm 1\right)=\frac{1}{2}$, then for all $\beta>0$ we have

$$
E\left[\exp \left(\beta \sup _{n}\left|\frac{\varepsilon_{1}+\cdots+\varepsilon_{n}}{\sqrt{2 n L L n}}\right|^{2}\right)\right]<\infty .
$$

We will obtain (1.9) as an easy corollary of the main result of $\$ 3$, and applications to the empirical distribution function will be given in $\S 4$. Another aspect of the paper involves moments of the type $E\left(\exp \left(\beta\|S\|^{2}\right)\right)$ where $S=\Sigma_{j>1} X_{j}$ is a convergent series, and the $\left\{X_{j}\right\}$ satisfy certain conditions. Theorem 3.2 and its corollaries contain these results.

2. Some introductory lemmas, Here we will establish some estimates to be used in proving the results of $\$ 3$.

LEMMA 2.1. Let $(B, \mathfrak{B},\|\cdot\|)$ be a linear measurable space and assume $X_{1}, X_{2}, \ldots$ are independent $(B, \mathscr{B})$ valued random variables such that for some $\beta>0$,

$$
\sup _{j>1} E\left(\exp \left(\beta\left\|X_{j}\right\|^{2}\right)\right)<\infty .
$$

If $S_{n}=\sum_{j=1}^{n} b_{j} X_{j}$ where $\left\{b_{j}\right\}$ is a sequence of nonnegative constants, then there exists $\Lambda, 0<\Lambda<\infty$, such that for all $n \geqslant 1$ and all $h$ we have

$$
E\left(\exp \left(h\left\|S_{n}\right\|\right)\right)<\exp \left\{h E\left\|S_{n}\right\|+h^{2} \Lambda^{2} \sum_{j=1}^{n} b_{j}^{2}\right\} .
$$

Proof. To prove (2.2) set $Y_{k}=S_{n}-b_{k} X_{k}, E_{k} \eta=E\left(\eta \mid X_{1}, \ldots, X_{k-1}\right)$, $E_{n+1} \eta=\eta, E_{1} \eta=E \eta$. Then

$$
\left\|S_{n}\right\|=\sum_{k=1}^{n} \eta_{k}+E\left\|S_{n}\right\|
$$

where $\eta_{k}=E_{k+1}\left\|S_{n}\right\|-E_{k}\left\|S_{n}\right\|(k=1, \ldots, n)$. Hence

$$
\begin{aligned}
E\left(\exp \left(h\left\|S_{n}\right\|\right)\right) & =E\left(\exp \left\{h E\left\|S_{n}\right\|+h \sum_{k=1}^{n} \eta_{k}\right\}\right) \\
& =E\left[\exp \left\{h E\left\|S_{n}\right\|+h \sum_{k=1}^{n-1} \eta_{k}\right\} E_{n}\left(\exp \left\{h \eta_{n}\right\}\right)\right] .
\end{aligned}
$$

Furthermore, by the triangle inequality and the independence of $X_{1}, \ldots, X_{n}$ we have 


$$
\begin{aligned}
\eta_{k} & <E_{k+1}\left\|Y_{k}\right\|+E_{k+1}\left\|b_{k} X_{k}\right\|-E_{k}\left\|Y_{k}\right\|+E_{k}\left\|b_{k} X_{k}\right\| \\
& =b_{k}\left\|X_{k}\right\|+b_{k} E\left\|X_{k}\right\| \quad(k=1, \ldots, n),
\end{aligned}
$$

and similarly,

$$
\eta_{k}>-b_{k}\left\|X_{k}\right\|-b_{k} E\left\|X_{k}\right\| \quad(k=1, \ldots, n),
$$

since $E_{k+1}\left\|Y_{k}\right\|=E_{k}\left\|Y_{k}\right\|$ (because $Y_{k}$ is independent of $X_{k}$ ). Hence

$$
\left|\eta_{k}\right| \leqslant b_{k}\left(\left\|X_{k}\right\|+E\left\|X_{k}\right\|\right) \quad(k=1, \ldots, n)
$$

so

$$
\left|\eta_{k}\right|<2 b_{k} \max \left(\left\|X_{k}\right\|, R\right) \quad(k=1, \ldots, n),
$$

where $R \equiv \sup _{j} E\left\|X_{j}\right\|<\infty$ by (2.1). Using (2.4) and iterating we have the lemma proved if we show

$$
E_{k}\left(\exp \left(h \eta_{k}\right)\right)<\exp \left\{b_{k}^{2} h^{2} \Lambda^{2}\right\} \quad(k>1) .
$$

Now $E_{k}\left(\eta_{k}\right)=0$ and using (2.8) we obtain

$$
\begin{aligned}
E_{k}\left(\exp \left(h \eta_{k}\right)\right) & =1+\sum_{r=1}^{\infty} h^{r} E_{k}\left(\eta_{k}^{r}\right) \\
= & 1+\sum_{n=1}^{\infty}\left[\frac{h^{2 n} E_{k}\left(\eta_{k}^{2 n}\right)}{(2 n) !}+\frac{h^{2 n+1} E_{k}\left(\eta_{k}^{2 n+1}\right)}{(2 n+1) !}\right] \\
< & 1+\sum_{n=1}^{\infty}\left[\frac{\left(2 h b_{k}\right)^{2 n} E\left(Z_{k}^{2 n}\right)}{(2 n) !}+\frac{\left(2|h| b_{k}\right)^{2 n+1} E\left(Z_{k}^{2 n+1}\right)}{(2 n+1) !}\right]
\end{aligned}
$$

where $Z_{k}=\max \left(\left\|X_{k}\right\|, R\right)$.

Since (2.1) holds there exists a $\beta>0$ such that

$$
L \equiv \sup _{k} E\left(\exp \left(\beta Z_{k}^{2}\right)\right)<\infty,
$$

and hence we have for all $t>0$ that

$$
P\left(Z_{k}>t\right)<L \exp \left\{-\beta t^{2}\right\}
$$

Now if $W$ is a mean zero Gausian random variable with variance $\sigma^{2}$, then an elementary estimate implies

$$
\begin{aligned}
P(|W|>t) & =\sqrt{\frac{2}{\pi}} \frac{1}{\sigma} \int_{t}^{\infty} \exp \left(\frac{-s^{2}}{2 \sigma^{2}}\right) d s \\
& <\sqrt{\frac{2}{\pi}} \frac{\sigma}{t} \exp \left(\frac{-t^{2}}{2 \sigma^{2}}\right) \quad(t>0) .
\end{aligned}
$$

Taking $1 / \sigma^{2}<\beta$ we have, by combining (2.12) and (2.13), a finite number $t_{0}>0$ such that 


$$
P\left(Z_{k}>t\right)<\sqrt{\frac{2}{\pi}} \frac{1}{\sigma} \int_{t}^{\infty} \exp \left(\frac{-s^{2}}{2 \sigma^{2}}\right) d s \quad\left(t>t_{0}\right) .
$$

Hence by standard computations (see, for example, Lemma 4.2 of Some new results on central limit theorems for $C(S)$ valued random variables by $\mathrm{M}$. B. Marcus, Lecture Notes in Math., Vol. 526, Springer-Verlag, New York) we have

$$
\begin{aligned}
E\left(Z_{k}^{2 n}\right) & =\int_{0}^{\infty} P\left(Z_{k}^{2 n}>t\right) d t \\
& <t_{0}^{2 n}+2[(2 n-1)(2 n-3) \ldots 5 \cdot 3 \cdot 1] \sigma^{2 n} .
\end{aligned}
$$

and

$$
\begin{aligned}
& E\left(Z_{k}\right)^{2 n+1} \\
& \quad<t_{0}^{2 n+1}+\sqrt{2 / \pi}(2 n+1) \sigma^{2 n+1}[(2 n-2)(2 n-4) \ldots 4 \cdot 2 \cdot 1] .
\end{aligned}
$$

Thus for $2|h| b_{k}<1$ we have

$$
\begin{aligned}
& \frac{\left(2 h b_{k}\right)^{2 n} E\left(Z_{k}^{2 n}\right)}{(2 n) !}+\frac{\left(2|h| b_{k}\right)^{2 n+1} E\left(Z_{k}^{2 n+1}\right)}{(2 n+1) !} \\
& \quad<\frac{\left(2 h b_{k}\right)^{2 n}}{(2 n) !}\left[t_{0}^{2 n}+t_{0}^{2 n+1}+2[(2 n-1) \ldots 5 \cdot 3 \cdot 1] \sigma^{2 n}\right. \\
& \left.\quad+\sqrt{2 / \pi}[(2 n-2) \ldots 4 \cdot 2 \cdot 1] \sigma^{2 n+1}\right] \\
& \quad<\frac{\left(2 h b_{k}\right)^{2 n}}{(2 n) !}\left[2\left(\bar{t}_{0}\right)^{2 n+1}+2[(2 n-1) \ldots 5 \cdot 3 \cdot 1] \bar{\sigma}^{2 n+1}\right] \\
& <\frac{\left(2 h b_{k}\right)^{2 n}}{(2 n) !}[(2 n-1) \ldots 5 \cdot 3 \cdot 1]\left[\bar{\sigma}^{2}+\bar{t}_{0}^{2}\right]^{2 n} \cdot
\end{aligned}
$$

Inserting (2.16) into (2.10) we have for $2|h| b_{k} \leqslant 1$ that

$$
\begin{aligned}
E_{k}\left(\exp \left(h \eta_{k}\right)\right) & <1+\sum_{n=1}^{\infty} \frac{[(2 n-1) \ldots 5 \cdot 3 \cdot 1]}{(2 n) !}\left[2 h b_{k}\left(\bar{\sigma}^{2}+\bar{t}_{0}^{2}\right)\right]^{2 n} \\
& =\exp \left\{2 h^{2} b_{k}^{2}\left(\bar{\sigma}^{2}+\bar{t}_{0}^{2}\right)^{2}\right\} .
\end{aligned}
$$

If $2|h| b_{k}>1$ then by (2.7) and the definition of $Z_{k}$ we have 


$$
\begin{aligned}
& E_{k}\left(\exp \left(h \eta_{k}\right)\right) \leqslant E\left(e^{2|h| b_{k} Z_{k}}\right)=1+\sum_{n=1}^{\infty}\left(2|h| b_{k}\right)^{n} \frac{E\left(Z_{k}^{n}\right)}{n !} \\
& <1+\sum_{j=1}^{\infty} \frac{\left(2 h b_{k}\right)^{2 j}}{(2 j) !}\left\{2 j E\left(Z_{k}^{2 j-1}\right)+E\left(Z_{k}^{2 j}\right)\right\} \\
& <1+\sum_{j=1}^{\infty} \frac{\left(2 h b_{k}\right)^{2 j}}{(2 j) !}\left\{2 j t_{0}^{2 j-1}+2 j \sqrt{2 / \pi}[(2 j-1)(2 j-4) \ldots 4 \cdot 2] \sigma^{2 j-1}\right. \\
& \left.+t_{0}^{2 j}+2[(2 j-1) \ldots 5 \cdot 3] \sigma^{2 j}\right\} \\
& <1+\sum_{j=1}^{\infty} \frac{\left(2 h b_{k}\right)^{2 j}}{(2 j) !}\left[\bar{t}_{0}^{2 j}(j+1)+(j+1)[(2 j-1) \ldots 5 \cdot 3] \bar{\sigma}^{2 j}\right] \\
& \text { where } \bar{t}_{0}=\max \left(t_{0}, 2\right) \text { and } \bar{\sigma}=\max (\sigma, 2) \\
& <1+\sum_{j=1}^{\infty} \frac{\left(2 h b_{k}\right)^{2 j}[(2 j-1) \ldots 5 \cdot 3]\left[(2 \bar{\sigma})^{2}+\left(2 \bar{t}_{0}\right)^{2}\right]^{2 j}}{(2 j) !} \\
& \text { since } x^{2 j}>j+1 \text { for } x>2 \\
& =\exp \left\{\left(h^{2} / 2\right) 4 b_{k}^{2}\left[(2 \bar{\sigma})^{2}+\left(2 \bar{t}_{0}\right)^{2}\right]^{2}\right\}
\end{aligned}
$$

Combining (2.17) and (2.18) we have for all $h$ that

$$
E_{k}\left(\exp \left(h \eta_{k}\right)\right)<\exp \left\{h^{2} b_{k}^{2} \Lambda^{2}\right\}
$$

where $\Lambda=4\left[(2 \bar{\sigma})^{2}+\left(2 \overline{t_{0}}\right)^{2}\right]$. Returning to $(2.9)$ we see the lemma is proved.

LEMMA 2.2. Let $(B, \Re,\|\cdot\|)$ be a linear measurable space, and assume $X_{1}, X_{2}, \ldots$ are independent symmetric $(B, \mathscr{B})$ valued random variables such that

(a) $L \equiv \sup _{j} E\left\|X_{j}\right\|^{2}<\infty$, and

(b) For some sequence of positive constants $\left\{b_{j}\right\}$ we have $\left\{\sum_{j=1}^{n} b_{j} X_{j} / \sigma_{n}: n>1\right\}$ bounded in probability where $\sigma_{n}^{2} \equiv b_{1}^{2}$ $+\cdots+b_{n}^{2}$.

Then, we have

$$
\sup _{n} E\left\|\sum_{j=1}^{n} b_{j} X_{j} / \sigma_{n}\right\|<\infty .
$$

Proof. Set $S_{n}=\sum_{j=1}^{n} b_{j} X_{j}$ for $n \geqslant 1$. Then from [7] or [5, Lemma 5.4] we 
have that

$$
\frac{1}{3} \int_{0}^{\infty} P\left(\left\|S_{n}\right\|>t\right) d t<\int_{0}^{\infty} P\left(N_{n}>t\right) d t+4 \int_{0}^{\infty}\left[P\left(\left\|S_{n}\right\|>t\right)\right]^{2} d t
$$

where $N_{n}=\sup _{1<j<n} b_{j}\left\|X_{j}\right\|$. Pick $A>0$ so that $\sup _{n>1} P\left(\left\|S_{n}\right\|>A \sigma_{n}\right) \leqslant$ 1/24. Then

$$
\frac{1}{3} \int_{A \sigma_{n}}^{\infty} P\left(\left\|S_{n}\right\|>t\right) d t \leqslant 5 A \sigma_{n}+\int_{A \sigma_{n}}^{\infty} P\left(N_{n}>t\right) d t+\frac{1}{6} \int_{A \sigma_{n}}^{\infty} P\left(\left\|S_{n}\right\|>t\right) d t
$$

and hence

$$
\frac{1}{6} \int_{0}^{\infty} P\left(\left\|S_{n}\right\|>t\right) d t<6 A \sigma_{n}+\int_{A \sigma_{n}}^{\infty} P\left(N_{n}>t\right) d t
$$

Now

$$
P\left(N_{n}>t\right)<\sum_{j=1}^{n} P\left(\left\|b_{j} X_{j}\right\|>t\right) \leqslant \sum_{j=1}^{n} b_{j}^{2} E\left\|X_{j}\right\|^{2} / t^{2}<\frac{L \sigma_{n}^{2}}{t^{2}},
$$

and hence

$$
\int_{A \sigma_{n}}^{\infty} P\left(N_{n}>t\right) d t \leqslant L \sigma_{n}^{2} \int_{A \sigma_{n}}^{\infty} t^{-2} d t=\frac{L \sigma_{n}}{A} .
$$

Combining (2.23) and (2.24) we have (2.21) so the lemma is proved.

3. Some exponential moments. We will prove several theorems regarding exponential moments of random variables of the form given in (1.2) as well as indicate some results for random series.

THEOREM 3.1. Let $(B, \mathscr{B},\|\cdot\|)$ be a linear measurable space and assume $X_{1}, X_{2}, \ldots$ are independent $(B, \mathscr{B})$ valued random variable such that

(a) $E\left(f\left(X_{j}\right)\right)=0$ for all $f \in F, j \geqslant 1$,

(b) $\sup _{j>1} E\left(\exp \left(\beta\left\|X_{j}\right\|^{2}\right)\right)<\infty$ for some $\beta>0$, and

(c) for some sequence of positive constants $\left\{b_{j}\right\}$ we have

(i) $\sigma_{n}^{2} \equiv b_{1}^{2}+\cdots+b_{n}^{2} \rightarrow \infty$ as $n \rightarrow \infty$,

(ii) $b_{n}^{2} / \sigma_{n}^{2} \rightarrow 0$ as $n \rightarrow \infty$, and

(iii) $\left\{\sum_{j=1}^{n} b_{j} X_{j} / \sigma_{n}: n \geqslant 1\right\}$ is bounded in probability.

If $S_{n}=\sum_{j=1}^{n} b_{j} X_{j}$ and $a_{n}=\sqrt{2 \sigma_{n}^{2} L L \sigma_{n}^{2}}$, then there exists $\beta_{0}>0$ such that $\beta<\beta_{0}$ implies

$$
E\left[\exp \left\{\beta \sup _{n}\left\|\frac{S_{n}}{a_{n}}\right\|^{2}\right\}\right]<\infty
$$

Furthermore, if (3.1)(b) holds for all $\beta>0$, then (3.2) holds for all $\beta>0$. 
REMARK. If (3.2) holds for all $\beta>0$, then it is obvious that (3.1)(b) holds for all $\beta>0$. The interesting thing is that the converse is also true under the reasonable assumption that $\left\{S_{n} / \sigma_{n}\right\}$ is bounded in probability. This, for example, is always the case if $B$ is a type 2 Banach space (see Corollary 3.1).

Proof. First assume the $\left\{X_{j}\right\}$ are symmetric, and define for $r=1,2, \ldots$,

$$
M_{r}=\sup _{n>r}\left\|S_{n} / a_{n}\right\| \text {. }
$$

First we will show that for every $\beta>0$ there exists an $r(\beta)$ such that $r \geqslant r(\beta)$ implies

$$
E\left(\exp \left(\beta M_{r}^{2}\right)\right)<\infty
$$

Once (3.4) is established, then (3.1)(b) easily implies that there exists a $\beta_{0}>0$ such that $\beta<\beta_{0}$ implies

$$
E\left[\exp \left[\beta \sup _{n}\left\|\frac{S_{n}}{a_{n}}\right\|^{2}\right]\right)<\infty .
$$

In fact, if (3.1)(b) holds for all $\beta>0$, then (3.4) yields (3.5) for all $\beta>0$ and the theorem is proved if the $\left\{X_{j}\right\}$ are symmetric. We will remove the symmetry assumption at the end of the proof and hence our aim now is to verify (3.4).

Set $n_{k}=\min \left\{j: \sigma_{j}^{2} \geqslant 2^{k}\right\}(k \geqslant 2)$ with $n_{1} \equiv 1$. Further, put $k(r)=\max \{j$ : $\left.n_{j}<r\right\}$ for $r \geqslant 1$. Then by using Levy's maximal inequality we have

$$
\begin{aligned}
P\left(M_{r} \geqslant \lambda\right) & <\sum_{k>k(r)+1} P\left(\max _{n_{k-1}<n<n_{k}} \frac{\left\|S_{n}\right\|}{a_{n}}>\lambda\right) \\
& <\sum_{k>k(r)+1} 2 P\left(\left\|S_{n_{k}}\right\|>\lambda a_{n_{k-1}}\right) \\
& =2 \sum_{k>k(r)+1} P\left(\frac{\left\|S_{n_{k}}\right\|}{2 \sigma_{n_{k}}}>\frac{\lambda \sigma_{n_{k-1}}}{2 \sigma_{n_{k}}} \sqrt{2 L L \sigma_{n_{k-1}}^{2}}\right) \\
& <2 \sum_{k>k(r)+1} P\left(\frac{\left\|S_{n_{k}}\right\|}{2 \sigma_{n_{k}}}>\frac{\lambda}{8} \sqrt{2 L L 2^{k-1}}\right)
\end{aligned}
$$

provided $r$ is sufficiently large so that $k \geqslant k(r)+1$ implies $\sigma_{n_{k-1}} / \sigma_{n_{k}}>1 / 4$. Such an $r$ exists since (3.1)(c)(i), (ii) implies that $\sigma_{n_{k-1}} / \sigma_{n_{k}} \rightarrow 1 / 2$ as $k \rightarrow \infty$.

Now by Lemma 2.1 we have $\Lambda, 0 \leqslant \Lambda<\infty$, such that for all $n>1$ and $h$,

$$
E\left(\exp \left(h\left\|S_{n}\right\|\right)\right) \leqslant \exp \left\{h E\left\|S_{n}\right\|+h^{2} \Lambda^{2} \sum_{j=1}^{n} b_{j}^{2}\right\}
$$

Hence 


$$
\begin{aligned}
P\left(\left\|S_{n}\right\| / 2 \sigma_{n}>t\right) & <\exp \{-h t\} E\left(\exp \left(h\left\|S_{n}\right\| / 2 \sigma_{n}\right)\right) \\
& <\exp \left\{-h t+\frac{h}{2 \sigma_{n}} E\left\|S_{n}\right\|+\frac{h^{2} \Lambda^{2}}{4 \sigma_{n}^{2}} \sum_{j=1}^{n} b_{j}^{2}\right\} \\
& =\exp \left\{-h\left(t-E\left\|S_{n}\right\| / 2 \sigma_{n}\right)+h^{2} \Lambda^{2} / 4\right\} .
\end{aligned}
$$

Minimizing (3.8) as a function of $h$ we have

$$
P\left(\left\|S_{n}\right\| / 2 \sigma_{n}>t\right)<\exp \left\{-\left(t-E\left\|S_{n}\right\| / 2 \sigma_{n}\right)^{2} / \Lambda^{2}\right\} .
$$

By Lemma 2.2 we have $\sup _{n} E\left\|S_{n}\right\| / \sigma_{n} \leqslant C<\infty$, so for $t>C$ we have from (3.9) that

$$
P\left(\left\|S_{n}\right\| / 2 \sigma_{n} \geqslant t\right) \leqslant \exp \left\{-t^{2} / 4 \Lambda^{2}\right\} .
$$

Substituting (3.10) into (3.6) we have for all $\lambda$ such that $\inf _{k>k(r)+1}(\lambda / 8) \sqrt{2 L L 2^{k-1}}>C$ that

$$
P\left(M_{r} \geqslant \lambda\right)<2 \sum_{k>k(r)+1} \exp \left\{-\frac{\lambda^{2} L L 2^{k-1}}{256 \Lambda^{2}}\right\} .
$$

Fix $\beta>0$ and choose $\alpha$ so that $(\log \alpha) / \beta>\max \left(64 C^{2}, 512 \Lambda^{2}, 2\right)$, and $r(\beta)$ such that $r>r(\beta)$ and $k>k(r)+1$ implies

$$
\begin{aligned}
& \text { (a) } \sigma_{n_{k-1}} / \sigma_{n_{k}}>1 / 4 \text { (and hence (3.6) and (3.11) hold), and } \\
& \text { (b) } L L 2^{k-1} / 256 \Lambda^{2}-\beta>\beta \text {. }
\end{aligned}
$$

Then by (3.11) we have

$$
\begin{aligned}
E\left(\exp \left(\beta M_{r}^{2}\right)\right) & =\int_{0}^{\infty} P\left(\exp \left(\beta M_{r}^{2}\right)>t\right) d t \\
& <\alpha+\int_{\alpha}^{\infty} P\left(M_{r}>\left(\frac{\log t}{\beta}\right)^{1 / 2}\right) d t \\
& =\alpha+\int_{\sqrt{(\log \alpha) / \beta}}^{\infty} P\left(M_{r}>s\right) 2 \beta s \exp \left\{\beta s^{2}\right\} d s \\
& <\alpha+2 \sum_{k>k(r)+1} \int_{\sqrt{(\log \alpha) / \beta}}^{\infty} \exp \left\{-s^{2}\left(\frac{L L 2^{k-1}}{256 \Lambda^{2}}-\beta\right)\right\} 2 \beta s d s \\
& <\alpha+2 \sum_{k>k(r)+1} \exp \left\{-\Lambda_{k} \log \alpha\right\} / \Lambda_{k} \\
& <\alpha+2 \sum_{k>k(r)+1} \frac{1}{[(k-1) L 2]^{\gamma}} \quad \text { where } \Lambda_{k}=\beta^{-1}\left[L L 2^{k-1} / 256 \Lambda^{2}-\beta\right]>1 \\
& <\infty \quad \text { since }(\log \alpha) / \beta 256 \Lambda^{2}>2 .
\end{aligned}
$$


Thus the theorem holds if $\left\{X_{j}\right\}$ is symmetric. To handle the situation when $\left\{X_{j}\right\}$ is not symmetric we introduce a sequence $\left\{Z_{j}: j>1\right\}$ which has the same distribution as $\left\{X_{j}\right\}$ and independent of $\left\{X_{j}\right\}$. Letting $E_{1}\left(E_{2}\right)$ denote expectations with respect to $\left\{X_{j}\right\}\left(\left\{Z_{j}\right\}\right)$, then by the previous case we have for the relevant $\beta>0$ that

$$
\begin{aligned}
& \infty>E_{1} E_{2}\left(\exp \left\{\beta \sup _{n}\left\|\sum_{j=1}^{n} b_{j}\left(X_{j}-Z_{j}\right) / a_{n}\right\|^{2}\right\}\right) \\
& >E_{1}\left\{\exp \left\{\beta E_{2} \sup _{n}\left\|\sum_{j=1}^{n} b_{j}\left(X_{j}-Z_{j}\right) / a_{n}\right\|^{2}\right\}\right\} \\
& \text { by Jensen's inequality } \\
& >E_{1}\left[\exp \left\{\beta \sup _{n} \sup _{f \in F}\left|\sum_{j=1}^{n} b_{j}\left[f\left(X_{j}\right)-E_{2} f\left(Z_{j}\right)\right] / a_{n}\right|^{2}\right\}\right] \\
& \text { by (1.1) and Jensen's inequality applied to } E_{2} \\
& \geqslant E_{1}\left[\exp \left\{\beta \sup _{n}\left\|\sum_{j=1}^{n} b_{j} X_{j} / a_{n}\right\|^{2}\right\}\right] \\
& \text { since } E_{2} f\left(Z_{j}\right)=0 \text { for all } f \in F \text {. }
\end{aligned}
$$

Thus the theorem is proved.

Corollary 3.1. Let $(R, \mathscr{B},\|\cdot\|)$ be a linear measurable space and assume $X_{1}, X_{2}, \ldots$ are i.i.d. $(B, \mathscr{B})$ valued random variables such that

(a) $E f\left(X_{i}\right)=0$ for all $f \in F$,

(b) $E\left(\exp \left(\beta\left\|X_{1}\right\|^{2}\right)\right)<\infty$ for some $\beta>0$, and

(c) $\left\{S_{n} / \sqrt{n}: n \geqslant 1\right\}$ is bounded in probability where $S_{n}=$ $\sum_{j=1}^{n} X_{j}$.

If $a_{n}=\sqrt{2 n L L n}$, then there exists $\beta_{0}>0$ such that $\beta<\beta_{0}$ implies

$$
E\left[\exp \left\{\beta \sup _{n}\left\|\frac{S_{n}}{a_{n}}\right\|^{2}\right\}\right)<\infty .
$$

Furthermore, if (3.15)(b) holds for all $\beta>0$, then (3.16) also holds for all $\beta>0$.

Proof. Immediate from Theorem 3.1. 
Corollary 3.2. Let $B$ denote a real separable Banach space of type 2. Let $X_{1}, X_{2}, \ldots$ be i.i.d. B-valued random variables such that

$$
\begin{aligned}
& \text { (a) } E X_{1}=0 \text {, and } \\
& \text { (b) } E\left(\exp \left(\beta\left\|X_{1}\right\|^{2}\right)\right)<\infty \text { for some } \beta>0 \text {. }
\end{aligned}
$$

Then the conclusions of Corollary 3.1 hold.

Proof. $E X_{1}=0$ easily implies (3.15)(a), and since $B$ is of type 2 we have a constant $A>0$ such that for all $n$,

$$
E\left\|S_{n}\right\|<\left(E\left\|S_{n}\right\|^{2}\right)^{1 / 2}<\left(A \sum_{j=1}^{n} E\left\|X_{j}\right\|^{2}\right)^{1 / 2}=A^{1 / 2} n^{1 / 2}\left(E\left\|X_{1}\right\|^{2}\right)^{1 / 2} .
$$

Thus $\sup _{n} E\left\|S_{n}\right\| / \sqrt{n}<\infty$ and hence (3.15)(c) holds, so the corollary is proved.

For the next corollary recall the linear measurable space $(D[0, T], \mathscr{B}$, $\|\cdot\|)$ defined in $\$ 1$.

COROLlaRY 3.3. If $X_{1}, X_{2}, \ldots$ are i.i.d. $(D[0, T], \mathscr{B})$ valued random variables such that

(a) $E X_{1}(t)=0$ for all $t \in[0, T]$,

(b) $E\left(\exp \left(\beta\left\|X_{1}\right\|^{2}\right)\right)<\infty$ for some $\beta>0$, and

(c) $\left\{X_{j}(t): 0<t<T\right\}$ is a martingale on $[0, T]$ for $j \geqslant 1$,

then there exists $a \beta_{0}>0$ such that $\beta \leqslant \beta_{0}$ implies

$$
E\left[\exp \left[\beta \sup _{n}\left\|\frac{S_{n}}{a_{n}}\right\|^{2}\right]\right)<\infty
$$

where $S_{n}=X_{1}+\cdots+X_{n}$ and $a_{n}=\sqrt{2 n L L n}$.

REMARK. Of course, if (3.19)(b) holds for all $\beta>0$ then (3.20) holds for all $\beta>0$.

Proof. This follows from Corollary 3.1 provided we show that (3.19)(c) implies (3.15)(c). Since the norm $\|\cdot\|$ is the supremum norm and $S_{n}(t) / \sqrt{n}$ is a martingale in $t, 0<t<T$, we have by the maximal inequality that

$$
\begin{aligned}
P\left(\left\|S_{n} / \sqrt{n}\right\| \geqslant \lambda\right) & \leqslant \lambda^{-1} E\left(\left|S_{n}(T) / \sqrt{n}\right|\right)<\left(E\left|S_{n}(T)\right|^{2}\right)^{1 / 2} / \lambda n^{1 / 2} \\
& =\lambda^{-1}\left(E\left|X_{1}(T)\right|^{2}\right)^{1 / 2}=O(1 / \lambda) .
\end{aligned}
$$

Hence (3.15)(c) holds and the corollary is proved.

LEMMA 3.1. Let $(B, \mathscr{B},\|\cdot\|)$ be a linear measurable space, and assume $X_{1}, X_{2}, \ldots$ are independent symmetric $(B, \mathscr{B})$ valued random variables such 
that

(a) $L \equiv \sup _{j} E\left\|X_{j}\right\|^{2}<\infty$,

(b) for some sequence of positive constants $\left\{b_{j}\right\}$ we have $\sum_{j=1}^{\infty} b_{j}^{2}<\infty$ and $\left\{\sum_{j=1}^{n} b_{j} X_{j} ; n>1\right\}$ is stochastically bounded.

Then

$$
\sup _{n} E\left\|\sum_{j=1}^{n} b_{j} X_{j}\right\|<\infty .
$$

Proof. Let $S_{n}=\sum_{j=1}^{n} b_{j} X_{j}$ and $N_{n}=\sup _{1<j<n}\left\|b_{j} X_{j}\right\|$ for $n>1$. Since $\left\{S_{n}\right.$ : $n>1\}$ is stochastically bounded there exists $A>0$ such that $\sup _{n} P\left(\left\|S_{n}\right\|>A\right)<\frac{1}{24}$. Hence by the reasoning used to establish Lemma 2.2 we have

$$
E\left\|S_{n}\right\|<36 A+6 \int_{A}^{\infty} P\left(N_{n}>t\right) d t
$$

Now

$$
\begin{aligned}
P\left(N_{n}>t\right) & <\sum_{j=1}^{n} P\left(\left\|b_{j} X_{j}\right\|>t\right) \\
& <\sum_{j=1}^{n} b_{j}^{2} E\left\|X_{j}\right\|^{2} / t^{2}<L \sum_{j=1}^{\infty} b_{j}^{2} / t^{2} .
\end{aligned}
$$

Letting $C=L \sum_{j=1}^{\infty} b_{j}^{2}$ we have $\sup _{n} E\left\|S_{n}\right\|<36 A+6 C / A$ and the lemma is proved.

THEOREM 3.2. Let $(B, \mathscr{B},\|\cdot\|)$ be a linear measurable space and assume $X_{1}, X_{2}, \ldots$ are independent $(B, \mathscr{B})$ valued random variables such that

(a) $E f\left(X_{j}\right)=0$ for all $f \in F$ and $j>1$,

(b) $\sup _{j>1} E\left(\exp \left(\beta\left\|X_{j}\right\|^{2}\right)\right)<\infty$ for some $\beta>0$, and

(c) the sequence of partial sums $\left\{S_{n} \equiv \sum_{j=1}^{n} b_{j} X_{j}, n \geqslant 1\right\}$ is stochastically bounded.

If $\Sigma_{j>1} b_{j}^{2}<\infty$, then there exists $\beta_{0}>0$ such that $\beta<\beta_{0}$ implies

$$
E\left(\exp \left(\beta \sup _{n}\left\|S_{n}\right\|^{2}\right)\right)<\infty \text {. }
$$

Furthermore, if (3.22)(b) holds for all $\beta>0$, then (3.23) also holds for all $\beta>0$.

Proof. First assume $X_{1}, X_{2}, \ldots$ are symmetric. Then Levy's maximal inequality and (3.22)(c) easily imply that $P\left(\sup _{n}\left\|S_{n}\right\|<\infty\right)=1$.

In view of (3.22)(b) the theorem is proved if for all $\beta>0$ there exists an $r(\beta)$ such that $r>r(\beta)$ implies 


$$
E\left(\exp \left(\beta \sup _{n>r}\left\|S_{n}-S_{r}\right\|^{2}\right)\right)<\infty
$$

Now

$$
P\left(\sup _{n>r}\left\|S_{n}-S_{r}\right\|>\lambda\right)=\lim _{N \rightarrow \infty} P\left(\sup _{r<n<N}\left\|S_{n}-S_{r}\right\|>\lambda\right),
$$

and hence by Levy's inequality

$$
P\left(\sup _{n>r}\left\|S_{n}-S_{r}\right\|>\lambda\right)<2 \lim _{N \rightarrow \infty} P\left(\left\|S_{N}-S_{r}\right\|>\lambda\right) .
$$

Now Lemma 2.1 implies

$$
\begin{aligned}
& P\left(\left\|S_{N}-S_{r}\right\|>\lambda\right)<\exp \{-h \lambda\} E\left(\exp \left(h\left\|S_{N}-S_{r}\right\|\right)\right) \\
& <\exp \left\{-h\left(\lambda-E\left\|S_{N}-S_{r}\right\|\right)+h^{2} \Lambda^{2} \sum_{j=r+1}^{N} b_{j}^{2}\right\} \\
& <\exp \left\{-\left(\lambda-E\left\|S_{N}-S_{r}\right\|\right)^{2} /\left(4 \Lambda^{2} \sum_{j=r+1}^{N} b_{j}^{2}\right)\right\} \\
& \text { by minimizing the function }
\end{aligned}
$$

by minimizing the function of $h$

By Lemma 3.1 we have $\sup _{n} E\left\|S_{n}\right\|<\infty$; so combining (3.25) and (3.26) we have for $\lambda / 2>\sup _{N>r} E\left\|S_{N}-S_{r}\right\|$ that

$$
P\left(\sup _{n>r}\left\|S_{n}-S_{r}\right\|>\lambda\right)<2 \exp \left\{-\lambda^{2} /\left(16 \Lambda^{2} \sum_{j=r+1}^{\infty} b_{j}^{2}\right)\right\} .
$$

Hence given $\beta>0$ we have

$$
\begin{aligned}
& E\left(\exp \left\{\beta \sup _{n>r}\left\|S_{n}-S_{r}\right\|^{2}\right\}\right)=\int_{0}^{\infty} P\left(\exp \left(\beta \sup _{n>r}\left\|S_{n}-S_{r}\right\|^{2}\right)>t\right) d t \\
& <\alpha+\int_{\alpha}^{\infty} P\left(\sup _{n>r}\left\|S_{n}-S_{r}\right\|>\left(\frac{\log t}{\beta}\right)^{1 / 2}\right) d t \\
& <\alpha+\int_{\alpha}^{\infty} 2 \exp \left\{-\log t /\left(16 \Lambda^{2} \beta \sum_{j=r+1}^{\infty} b_{j}^{2}\right)\right\} d t \\
& \text { provided } \sqrt{(\log \alpha) / \beta}>2 \sup _{n>r} E\left\|S_{n}-S_{r}\right\| \\
& =\alpha+2 \int_{\alpha}^{\infty}\left(\frac{1}{t}\right)^{\left(16 \Lambda^{2} \beta \sum_{j=r+1}^{\infty} b_{j}^{2}\right)^{-1}} d t \\
& <\infty \text { if } 16 \Lambda^{2} \beta \sum_{j=r+1}^{\infty} b_{j}^{2}<1 .
\end{aligned}
$$


Since $\Sigma_{j>1} b_{j}^{2}<\infty$ there exists $r(\beta)$ such that $r>r(\beta)$ implies $16 \Lambda^{2} \beta \sum_{j=r+1}^{\infty} b_{j}^{2}<1$ so the theorem is proved in case $X_{1}, X_{2}, \ldots$, are symmetric.

If the sequence $\left\{X_{j}\right\}$ is not symmetric, then we proceed as in the proof of Theorem 3.2 and the theorem is proved.

The next result is related to [6, Theorem 6.1], [14], and [15].

COROLlary 3.4. Let $(B, \mathscr{B},\|\cdot\|)$ be a linear measurable space, $\left\{x_{j}: j>1\right\}$ elements in $B$, and $\left\{Y_{j}: j>1\right\}$ independent real-valued random variables such that

(a) $E Y_{j}=0(j>1)$ and

(b) $\sup _{j>1} E\left(\exp \left(\beta Y_{j}^{2}\right)\right)<\infty$ for some $\beta>0$.

If $S=\Sigma_{j \geq 1} Y_{j} x_{j}$ converges with probability one and $\Sigma_{j \geq 1}\left\|x_{j}\right\|^{2}<\infty$, then there exists $\beta_{0}>0$ such that $\beta<\beta_{0}$ implies

$$
E\left(\exp \left\{\beta\|S\|^{2}\right\}\right)<\infty \text {. }
$$

In fact, we have

$$
E\left[\exp \left\{\beta \sup _{n}\left\|\sum_{j=1}^{n} Y_{j} x_{j}\right\|^{2}\right]\right\}<\infty
$$

provided $\beta>0$ is sufficiently small. If (3.29)(b) holds for all $\beta>0$, then so does (3.31).

Proof. Let $b_{j}=\left\|x_{j}\right\|$ and define $X_{j}=Y_{j} x_{j} /\left\|x_{j}\right\|$ for $j>1$. Then Theorem 3.2 applies immediately so the result is proved.

For the next result we need the idea of a cotype 2-Banach space. We say a Banach space $B$ with norm $\|\cdot\|$ is of cotype 2 if for all sequences of mean zero independent $B$-valued random variables $X_{1}, X_{2}, \ldots$, we have a universal constant $A$ such that for all $n$,

$$
E\left\|X_{1}+\cdots+X_{n}\right\|^{2}>A \sum_{i=1}^{n} E\left\|X_{j}\right\|^{2} .
$$

Corollary 3.5. Let $(B,\|\cdot\|)$ be a cotype 2-Banach space and $\left\{Y_{j}\right\} a$ sequence of i.i.d. real-valued random variables such that (3.29) holds. If $S=\Sigma_{j \geqslant 1} Y_{j} x_{j}$ converges with probability one in $B$, then the conclusions of Corollary 3.4 hold.

Proof. If $Y_{j} \equiv 0$ there is nothing to prove so assume the contrary. Next observe that by Theorem 6.2 of [8] we have $E\|S\|^{2}<\infty$. Since $\left\{Y_{j}\right\}$ is an independent sequence with mean zero and $B$ is of cotype 2 we have for each $n$ that 


$$
E\|S\|^{2} \geqslant E\left\|\sum_{j=1}^{n} Y_{j} x_{j}\right\|^{2} \geqslant A E Y_{1}^{2} \sum_{j=1}^{n}\left\|x_{j}\right\|^{2} .
$$

Hence $\Sigma_{j>1}\left\|x_{j}\right\|^{2}<\infty$, and the result follows immediately from Corollary 3.4 .

The next result now follows immediately from Corollary 3.5 .

Corollary 3.6. Let $(B,\|\cdot\|)$ be a cotype 2-Banach space and $\left\{Y_{j}\right\} a$ sequence of $i . i . d$. real-valued random variables which are uniformly bounded. If $S=\sum_{j \geqslant 1} Y_{j} x_{j}$ converges with probability one, then

$$
E\left[\exp \left[\beta \sup _{n}\left\|\sum_{j=1}^{n} Y_{j} x_{j}\right\|^{2}\right]\right)<\infty \text { for all } \beta>0 .
$$

REMARK. Since the real numbers are cotype 2 the above results easily prove the following. If $S$ is a square integrable real-valued random variable with $L^{2}$-expansion $\Sigma_{j \geq 1} Y_{j} a_{j}$ where $\left\{Y_{j}\right\}$ is an i.i.d. sequence satisfying (3.29), then there exists a $\beta_{0}>0$ such that for $\beta \leqslant \beta_{0}$,

$$
E\left(\exp \left(\beta S^{2}\right)\right)<\infty \text {. }
$$

Of course, if (3.29)(b) holds for all $\beta>0$ then (3.24) also holds for all $\beta>0$. This generalizes the result $[18$, p. 215$]$ regarding Fourier expansions with respect to Rademacher functions.

4. An application to empirical distribution functions. The previous results easily apply to the multidemsional empirical distribution function, and it is this that we turn to now.

Let $X=\left(U_{1}, \ldots, U_{d}\right)$ be a random variable with values in $\mathbf{R}^{d}$. The distribution function $F$ of $X$ is defined as usual by

$$
F\left(x_{1}, \ldots, x_{d}\right)=P\left(U_{1}<x_{1}, \ldots, U_{d}<x_{d}\right) \quad\left(x_{1}, \ldots, x_{d}\right) \in \mathbf{R}^{d} .
$$

If $X_{1}, X_{2}, \ldots$ are independent random variables with distribution $F$ the empirical distribution function is given by

$$
\mathcal{E}_{n}(x)=\sum_{j=1}^{n} 1_{(-\infty, t]}\left(X_{j}\right) / n \quad\left(x \in \mathbf{R}^{d}, n>1\right)
$$

where $(-\infty, x]=\left\{\left(u_{1}, \ldots, u_{d}\right): u_{j} \leqslant x_{j}, 1 \leqslant j \leqslant d\right\}$ and $x=\left(x_{1}, \ldots, x_{d}\right)$. If $x=\left(x_{1}, \ldots, x_{d}\right)$ and $y=\left(y_{1}, \ldots, y_{n}\right)$ we write $x<y$ if $x_{j} \leqslant y_{j}(1<j<$ d).

Of course, $\left\{\delta_{n}(x): x \in \mathbf{R}^{d}, n \geqslant 1\right\}$ is a sequence of stochastic processes indexed by $\mathbf{R}^{d}$ and for each $x \in \mathbf{R}^{d}$,

$$
E\left(\xi_{n}(x)\right)=F(x) \text {. }
$$


By the law of large numbers we thus have

$$
\lim _{n}\left|\mathcal{E}_{n}(x)-F(x)\right|=0 \quad\left(x \in \mathbf{R}^{d}\right),
$$

and if $F$ is continuous it is easy to see that we actually have

$$
\lim _{n} \sup _{x \in \mathbb{R}^{d}}\left|\delta_{n}(x)-F(x)\right|=0 .
$$

If $d=1$, the distribution of $D_{n} \equiv \sup _{x}\left|\mathcal{E}_{n}(x)-F(x)\right|$ is the same for all continuous $F$, and Kolmogorov [12] first computed the limiting distribution of $n^{1 / 2} D_{n}$ as $n \rightarrow \infty$. Furthermore, Chung [2] gave a bound on the error term which showed that the law of the iterated logarithm

$$
P\left(\varlimsup_{n} \sqrt{n / 2 L L n} D_{n}=1 / 2\right)=1
$$

holds.

If $d>1$, the limiting distribution of $n^{1 / 2} D_{n}$ was proved to exist by Kiefer and Wolfowitz [10], and its form depends on $F$ unlike the case $d=1$. However, (4.4) holds for all $d>1$ provided $F$ is continuous, as proved by Kiefer [11]. In addition, functional laws of the iterated logarithm are also known in this setting [4], [17].

What we do here is examine the integrability of the random variable

$$
M=\sup _{n} \sqrt{n / 2 L L n} D_{n} .
$$

Before stating the integrability results for $M$ we need to define the linear measurable space $\left(D\left(\mathbf{R}^{d}\right), \mathscr{B},\|\cdot\|\right)$. That is, we call a function $x: \mathbf{R}^{d} \rightarrow \mathbf{R}^{1}$ a step function if $x$ is a finite linear combination of functions of the form

$$
t \rightarrow 1_{E_{1} \times \cdots \times E_{d}}(t)
$$

where $E_{j}$ is a left closed, right open subinterval of $\mathbf{R}^{1}$, and $1_{E}$ is the indicator function of the set $E$. Then $D\left(\mathbf{R}^{d}\right)$ is defined to be the uniform closure, in the space of all uniformly bounded functions from $\mathbf{R}^{d}$ to $\mathbf{R}^{1}$, of the linear subspace of step functions. A characterization of $D\left(\mathbf{R}^{d}\right)$ in terms of continuity properties can also be made. If $t \in \mathbf{R}^{d}$ and if, for $1<j<d, R_{j}$ is one of the relations $<$ and $\geqslant$, let $Q_{R_{1}, \ldots, R_{d}}(t)$ denote the quadrant $\left\{\left(s_{1}, \ldots, s_{d}\right) \in \mathbf{R}^{d}: s_{j} R_{j} t_{j}, 1 \leqslant j \leqslant d\right\}$. Then it is known that $x \in D\left(\mathbf{R}^{d}\right)$ iff for each $t \in \mathbf{R}^{d}$, we have $x_{Q} \equiv \lim _{s \rightarrow t, s \in Q} x(s)$ existing for each of the $2^{d}$ quadrants $Q=Q_{R_{1}, \ldots, R_{d}}(t)$, and $x(t)=x_{Q^{*}}$ for $Q^{*}=Q_{>} \ldots,>$. Hence we say the functions of $D\left(\mathbf{R}^{d}\right)$ are continuous from above (or the right), and have limits from below (or the left). The sigma-algebra $\mathscr{B}$ is the minimal $\sigma$-algebra making the maps $x \rightarrow x(t), t \in \mathbf{R}^{d}$, all measurable, and the norm $\|\cdot\|$ is the usual sup-norm given by

$$
\|x\|=\sup _{t \in \mathbf{R}^{d}}|x(t)| .
$$


That $\left(D\left(\mathbf{R}^{d}\right), \mathscr{B},\|\cdot\|\right)$ is actually a measurable linear space follows easily from the fact that an element $x \in D\left(\mathbf{R}^{d}\right)$ is uniquely determined by its values on any fixed countable dense subset of $\mathbf{R}^{d}$, and then emphasizing the ideas of [3].

Theorem 4.1. Let $X_{1}, X_{2}, \ldots$, be independent $\mathbf{R}^{d}$ valued random variables with common distribution function $F$. Then for all $\beta>0$ we have

$$
E\left(\exp \left\{\beta M^{2}\right\}\right)<\infty
$$

where $M$ is defined as in (4.5).

Proof. Setting $Y_{j}(t, \omega)=1_{(-\infty, t]}\left(X_{j}(\omega)\right)-F(t)$ for $j \geqslant 1$ and $t \in \mathbf{R}^{d}$ we have $\left\{Y_{j}\right\}$ a sequence of i.i.d. $\left(D\left(\mathbf{R}^{d}\right), \mathscr{B},\|\cdot\|\right)$ valued random variables which are uniformly bounded by one. Furthermore, we have

$$
M=\left\|\sum_{j=1}^{n} Y_{j} / \sqrt{2 n L L n}\right\|,
$$

and hence (4.6) follows immediately from Theorem 3.1 if we have

$$
\left\{\sum_{j=1}^{n} Y_{j} / \sqrt{n}: n>1\right\}
$$

bounded in probability in $\left(D\left(\mathbf{R}^{d}\right), \mathscr{B},\|\cdot\|\right)$. That the sequence $\left\{\sum_{j=1}^{n} Y_{j} / \sqrt{n}: n>1\right\}$ is bounded in probability follows immediately from $[11$, Theorem $1(\mathrm{~m})]$ so the proof is complete.

REMARK. Since $P(M<\infty)=1$, we easily see that

$$
\sup _{x \in \mathbf{R}^{d}}\left|\mathcal{E}_{n}(x)-F(x)\right|=O(\sqrt{L L n / n}),
$$

and this gives a rate of convergence for the empirical distribution $\mathcal{E}_{n}$ to $F$. One can prove more than (4.8) (provided $F$ is continuous), and this is done in [11, Theorem 2]. Furthermore, the integrability result in (4.6) could also be obtained directly from [11, Theorem $1(\mathrm{~m})$, but this would require a good bit of additional work. A result related to (4.8) when $d=1$ and $X_{1}, X_{2}, \ldots$, are independent, but possibly nonidentically distributed random variables, appears in [16].

\section{REFERENCES}

1. L. Breiman, Probability, Addison-Wesley, Reading, Mass., 1968. MR 37 \#4841.

2. K.-L. Chung, An estimate concerning the Kolmogorov limit distribution, Trans. Amer. Math. Soc. 67 (1949), 36-50. MR 11, 606.

3. P. J. Fernandez, On the weak convergence of random sums of independent random elements, $\mathrm{Ph}$. D. dissertation, submitted to the University of California, Berkeley, 1969.

4. H. Finkelstein, The law of the iterated logarithm for empirical distributions, Ann. Math. Statist. 42 (1971), 607-615. MR 44 \#4803. 
5. N. C. Jain, The central limit theorem in Banach space, (Proc. 1st Conf. on Probability in Banach Spaces, 1975), Lecture Notes in Math., Vol. 526, Springer-Verlag, Berlin and New York, 1976.

6. N. C. Jain and M. B. Marcus, Integrability of infinite sums of independent vector-valued random variables, Trans. Amer. Math. Soc. 212 (1975), 1-36. MR 52 \#6854.

7. J. Hoffman-Jørgensen, Sums of independent Banach space valued random variables, Studia Math. 52 (1974), 159-186. MR 50 \#8626.

8. Series No. 15, Matematisk Institut, Aarhus, Denmark, 1972-73.

9. Integrability of semi-norms, the 0-1 law, and the affine kernel for product measures, Aarhus Univ. Preprint Series No. 6, Matematisk Institut, Aarhus, Denmark, 1974-75.

10. J. Kiefer and J. Wolfowitz, On the deviations of the empiric distribution function of vector change variables, Trans. Amer. Math. Soc. 87 (1958), 173-186. MR 20 \#5519.

11. J. Kiefer, On large derivations of the empiric $\mathbf{D}$. F. of vector chance variables and a law of the iterated logarithm, Pacific J. Math. 11 (1961), 649-660. MR 24 \#A1732.

12. A. N. Kolmogorov, Sulla determinzione empirica di una legge di distribuzione, Ist. Ital. Atti. Giorn. 4 (1933), 88-91.

13. J. Kuelbs, A counterexample for Banach space valued random variables, Ann. Probability 4 (1976), 684-689.

14. W. Krakowiak, Comparison theorems for exponential moments of random series in Banach spaces (preprint).

15. S. Kwapien, $A$ theorem on the Rademacher series with vector valued coefficients (Proc. 1st Conf. on Probability in Banach Spaces, 1975), Lecture Notes in Math., vol. 526, Springer-Verlag。 Berlin and New York, 1976.

16. R. S. Singh, On the Glivenko-Cantelli theorem for weighted empiricals based on independent random variables, Ann. Probability 3 (1975), 371-374. MR 51 \#9173.

17. M. J. Wichura, Some Strassen-type laws of the iterated logarithm for multiparameter stochastic processes with independent increments, Ann. Probability 1 (1973), 272-296.

18. A. Zygmund, Trigonometric series. I, II, 2nd ed., Cambridge Univ. Press, London and New York, 1959. MR 21 \#6498.

DePartment of Mathematics, UNIVERSTTY OF Wisconsin, MAdison, Wisconsin 53706 